\title{
Transverse force tomography
}

Fatma P. Aslan, Matthias Burkardt, and Marc Schlegel@

Department of Physics, New Mexico State University, Las Cruces, New Mexico 88003-0001, USA

(Received 24 September 2019; published 27 November 2019)

While twist-2 GPDs allow for a determination of the distribution of partons on the transverse plane, twist-3 GPDs contain quark-gluon correlations that provide information about the average transverse color Lorentz force acting on quarks. We demonstrate how twist-3 GPDs can be used to provide transverse position information about that force.

DOI: 10.1103/PhysRevD.100.096021

\section{INTRODUCTION}

While twist-2 parton distribution functions (PDFs) provide information about the longitudinal momentum distribution of partons, two-dimensional Fourier transforms of twist-2 generalized parton distribution functions (GPDs) for a vanishing skewness parameter $\xi=0$ provide information on the longitudinal momentum distribution of partons in the transverse plane (impact parameter space) [1], i.e.,

$$
q\left(x, \mathbf{b}_{\perp}\right)=\int \frac{d^{2} \Delta_{\perp}}{(2 \pi)^{2}} e^{-i \mathbf{b}_{\perp} \cdot \mathbf{\Delta}_{\perp}} \mathrm{GPD}^{\mathrm{twt}-2}\left(x,-\Delta_{\perp}^{2}\right),
$$

where $q\left(x, \mathbf{b}_{\perp}\right)$ is the impact parameter parton distribution as a function of the separation $\left(\mathbf{b}_{\perp}\right)$ from the transverse center of momentum $\left(\mathbf{R}_{\perp} \equiv \sum_{i=q, g} \mathbf{r}_{\perp, i} x_{i}\right)$.

On the other hand, twist-3 distributions involve quarkgluon correlations that are not contained in twist- 2 distributions. Even though they do not have a single particle density interpretation like twist- 2 distributions, it has been shown that the $x^{2}$ moments of intrinsic twist-3 PDFs are related to the quark-gluon correlations which have a further interpretation as a force [2]. For example, the chirally even spin-dependent twist-3 parton distribution $g_{T}^{q}(x)$, defined as (see, e.g., Ref. [3])

$-M g_{T}^{q}(x)=\int_{-\infty}^{\infty} \frac{\mathrm{d} \lambda}{4 \pi} \mathrm{e}^{i \lambda x}\left\langle P, S_{T}\left|\bar{q}(0) \oiint_{T} \gamma_{5}[0 ; \lambda n] q(\lambda n)\right| P, S_{T}\right\rangle$,

where $P^{\mu}$ and $M$ are the nucleon's four momentum and mass, respectively, while $n^{\mu}$ is a light-cone vector with $n^{2}=0$

Published by the American Physical Society under the terms of the Creative Commons Attribution 4.0 International license. Further distribution of this work must maintain attribution to the author(s) and the published article's title, journal citation, and DOI. Funded by SCOAP ${ }^{3}$. and $n \cdot P=1$, and $S_{T}$ refers to the transverse nucleon polarization vector. Furthermore, the definition (2) contains quark fields $q$ and a Wilson line $[0 ; \lambda n]$ that ensures color gauge invariance. The twist- 3 PDF $g_{T}^{q}$ can be expressed as a sum of a piece that is determined entirely in terms of twist-2 helicity PDF $g_{1}^{q}(x)$ [Wandzura-Wilczek (WW) contribution] and an interaction dependent dynamical twist-3 term, $\bar{g}_{T}^{q}(x)$, which involves quark-gluon correlations [4],

$$
\begin{aligned}
g_{T}^{q}(x) & =g_{T}^{q, W W}(x)+\bar{g}_{T}^{q}(x), \\
g_{T}^{q, W W}(x) & =\int_{x}^{1} \frac{d y}{y} g_{1}^{q}(y) .
\end{aligned}
$$

The explicit form of $\bar{g}_{T}^{q}(x)$ in terms of quark-gluon correlations can be found in Eq. (46) of Ref. [3]. For simplicity, the contribution of a quark mass term has been neglected in Eq. (3).

The $x^{2}$ moment of the dynamical twist 3 term $\bar{g}_{T}^{q}(x)$ is called $d_{2}^{q} / 3$,

$$
\int_{-1}^{1} \mathrm{~d} x x^{2} \bar{g}_{T}^{q}(x)=\frac{d_{2}^{q}}{3}
$$

and can be related to the following local matrix element $[5,6],{ }^{1}$

$$
d_{2}^{q}=-\frac{1}{2 M\left(P^{+}\right)^{2} S^{x}}\left\langle P, S_{T}\left|\bar{q}(0) \gamma^{+} g G^{+y}(0) q(0)\right| P, S_{T}\right\rangle,
$$

where a special choice for the vectors $P^{\mu}=P^{+}(1,0$, $0,1) / \sqrt{2}$ and $n^{\mu}=(1,0,0,-1) /\left(\sqrt{2} P^{+}\right)$was assumed. Furthermore, $S^{x}$ is the nucleon polarization in the $\hat{x}$ direction and $G^{+y}$ is the gluon field strength tensor.

\footnotetext{
${ }^{1}$ Note that a-sign appears in Eq. (5), which was missing in Ref. [2].
} 
Some experimental information on the twist-3 parton distribution $g_{T}$, and consequently on the second moment $d_{2}^{q}$, may be obtained from the $g_{2}$ structure functions in polarized deep-inelastic lepton-nucleon (DIS) scattering.

As described in Ref. [2], the local matrix element, $\left\langle P, S_{T}\left|\bar{q}(0) \gamma^{+} g G^{+y}(0) q(0)\right| P, S_{T}\right\rangle$, appearing in Eq. (5), has a semiclassical interpretation as the average transverse color Lorentz force acting on the struck quark in a DIS experiment at the instant after it has been hit by the virtual photon, i.e.,

$F^{q, y}(0) \equiv-\frac{1}{\sqrt{2} P^{+}}\left\langle P, S_{T}\left|\bar{q}(0) \gamma^{+} g G^{+y}(0) q(0)\right| P, S_{T}\right\rangle$.

Comparing Eqs. (5) and (6) suggests a connection between $d_{2}$ and this force. In particular,

$$
F^{q, y}(0)=\sqrt{2} M P^{+} S^{x} d_{2}^{q} .
$$

The two-dimensional Fourier transform of the twist-2 GPDs lead to impact paramater space distributions. On the other hand the $x^{2}$ moments of the intrinsic twist-3 PDF $g_{T}$ can be related to the transverse color Lorentz force. The main purpose of this paper is to combine these two ideas and explore a physical interpretation for the Fourier transform of $x^{2}$ moments of intrinsic twist-3 GPDs as the distribution of the average transverse color Lorentz force on the transverse plane.

\section{COLOR LORENTZ FORCE DISTRIBUTION IN THE TRANSVERSE PLANE}

By taking the second moment of an intrinsic twist-3 PDF one can express this object in terms of a matrix element of a local operator that includes the covariant derivative $n \cdot D=n \cdot \partial-i g n \cdot A=\left(\partial^{+}-i g A^{+}\right) / P^{+}$acting on quark fields. In particular, the antisymmetric combination is relevant, $\bar{q} \stackrel{\leftrightarrow}{D^{+}} q=\frac{1}{2}\left(\bar{q}\left(D^{+} q\right)-\left(D^{+} \bar{q}\right) q\right)$. For example the $x^{2}$ moment of the chirally even spin-dependent twist-3 parton distribution, $g_{T}^{q}(x)$, can be written as

$$
\begin{aligned}
& \int_{-1}^{1} \mathrm{~d} x x^{2} g_{T}^{q}(x) \\
& \quad=-\frac{1}{2 S^{x} M P^{+2}}\left\langle P, S_{T}\left|\bar{q}(0) \gamma^{x} \gamma_{5}\left(\stackrel{\leftrightarrow}{D}^{+}\right)^{2} q(0)\right| P, S_{T}\right\rangle .
\end{aligned}
$$

If we compare this local matrix element with the second moment of the rhs of Eq. (3), we find

$$
\begin{aligned}
\left\langle P, S_{T}\left|\bar{q}(0) \gamma^{x} \gamma_{5}\left(\stackrel{\leftrightarrow}{D^{+}}\right)^{2} q(0)\right| P, \lambda\right\rangle & \\
= & -2 S^{x} M P^{+2} \int_{-1}^{1} \mathrm{~d} x x^{2} g_{T}^{q, W W}(x) \\
& +\frac{1}{3}\left\langle P, \lambda\left|\bar{q}(0) \gamma^{+} g G^{+y}(0) q(0)\right| P, \lambda\right\rangle
\end{aligned}
$$

The main idea is to generalize the forward matrix elements in Eq. (9) to nonforward matrix elements-which is possible if there is no momentum transfer in the $n$-direction from the initial state to the final state; or, in other words, the GPD skewness parameter $\xi$ vanishes, i.e., $\xi=0$. In this way, additional information on the position dependence of the transverse color Lorentz force can be obtained. The nonforward generalization of Eq. (9) has the following form,

$$
\begin{aligned}
\left\langle p^{\prime}, \lambda^{\prime}\right| & \left.\bar{q}(0) \gamma^{x} \gamma_{5}\left(\stackrel{\leftrightarrow}{D}^{+}\right)^{2} q(0)|p, \lambda\rangle\right|_{\xi=0} \\
= & \int_{-1}^{1} \mathrm{~d} x x^{2} \mathrm{GPD}^{\mathrm{WW}}\left(x, 0,-\Delta_{\perp}^{2}\right) \\
& \quad+\left.\frac{1}{3}\left\langle p^{\prime}, \lambda^{\prime}\left|\bar{q}(0) \gamma^{+} g G^{+y}(0) q(0)\right| p, \lambda\right\rangle\right|_{\xi=0} .
\end{aligned}
$$

In analogy to Eq. (3) we expect the WW term in (10) to consist of twist-2 GPDs only. The second term on the rhs of (10) is the nonforward matrix element of the same operator that provides the average force in Eq. (6). This suggests that the transverse force distribution can be studied by the $x^{2}$ moments of twist-3 GPDs. Using transversely localized states $\left|P^{+}, \mathbf{R}_{\perp}=0, \lambda\right\rangle \equiv \mathcal{N} \int d^{2} p_{\perp}\left|P^{+}, \mathbf{p}_{\perp}, \lambda\right\rangle$, where $\mathcal{N}$ is a normalization factor, the transverse force distribution can be defined as

$$
\begin{aligned}
\mathcal{F}_{\lambda^{\prime} \lambda}^{i}\left(\mathbf{b}_{\perp}\right) \equiv & -\frac{1}{\sqrt{2} P^{+}}\left\langle P^{+}, \mathbf{R}_{\perp}=0, \lambda^{\prime}\right| \bar{q}\left(\mathbf{b}_{\perp}\right) \gamma^{+} g G^{+i}\left(\mathbf{b}_{\perp}\right) \\
& \times q\left(\mathbf{b}_{\perp}\right)\left|P^{+}, \mathbf{R}_{\perp}=0, \lambda\right\rangle .
\end{aligned}
$$

Therefore, just as the Fourier transform of the twist-2 GPDs gives the longitudinal momentum distribution in the transverse plane in Eq. (1), the Fourier transform of the nonforward local matrix element (11) gives the distribution of the force in the transverse plane, i.e.,

$$
\mathcal{F}_{\lambda^{\prime} \lambda}^{i}\left(\mathbf{b}_{\perp}\right)=\int \frac{d^{2} \Delta_{\perp}}{(2 \pi)^{2}} e^{-i \mathbf{b}_{\perp} \cdot \boldsymbol{\Delta}_{\perp}} F_{\lambda^{\prime} \lambda}^{i}\left(\boldsymbol{\Delta}_{\perp}\right)
$$

with

$$
\begin{aligned}
F_{\lambda^{\prime} \lambda}^{i}\left(\boldsymbol{\Delta}_{\perp}\right)= & -\frac{1}{\sqrt{2} P^{+}}\left\langle P^{+}, \frac{\boldsymbol{\Delta}_{\perp}}{2}, \lambda^{\prime}\right| \bar{q}(0) \gamma^{+} g G^{+i}(0) \\
& \times q(0)\left|P^{+},-\frac{\boldsymbol{\Delta}_{\perp}}{2}, \lambda\right\rangle .
\end{aligned}
$$

\section{FORM FACTORS OF $\bar{q} G q$ CORRELATOR}

In order to gain further insight into the physical meaning of the impact parameter Lorentz-force matrix element (12) we parametrize the general matrix element,

$$
W^{\mu, \nu \lambda}\left(p, p^{\prime}\right)=\left\langle p^{\prime}, \lambda^{\prime}\left|\bar{q}(0) \gamma^{\mu} i g G^{\nu \lambda}(0) q(0)\right| p, \lambda\right\rangle,
$$



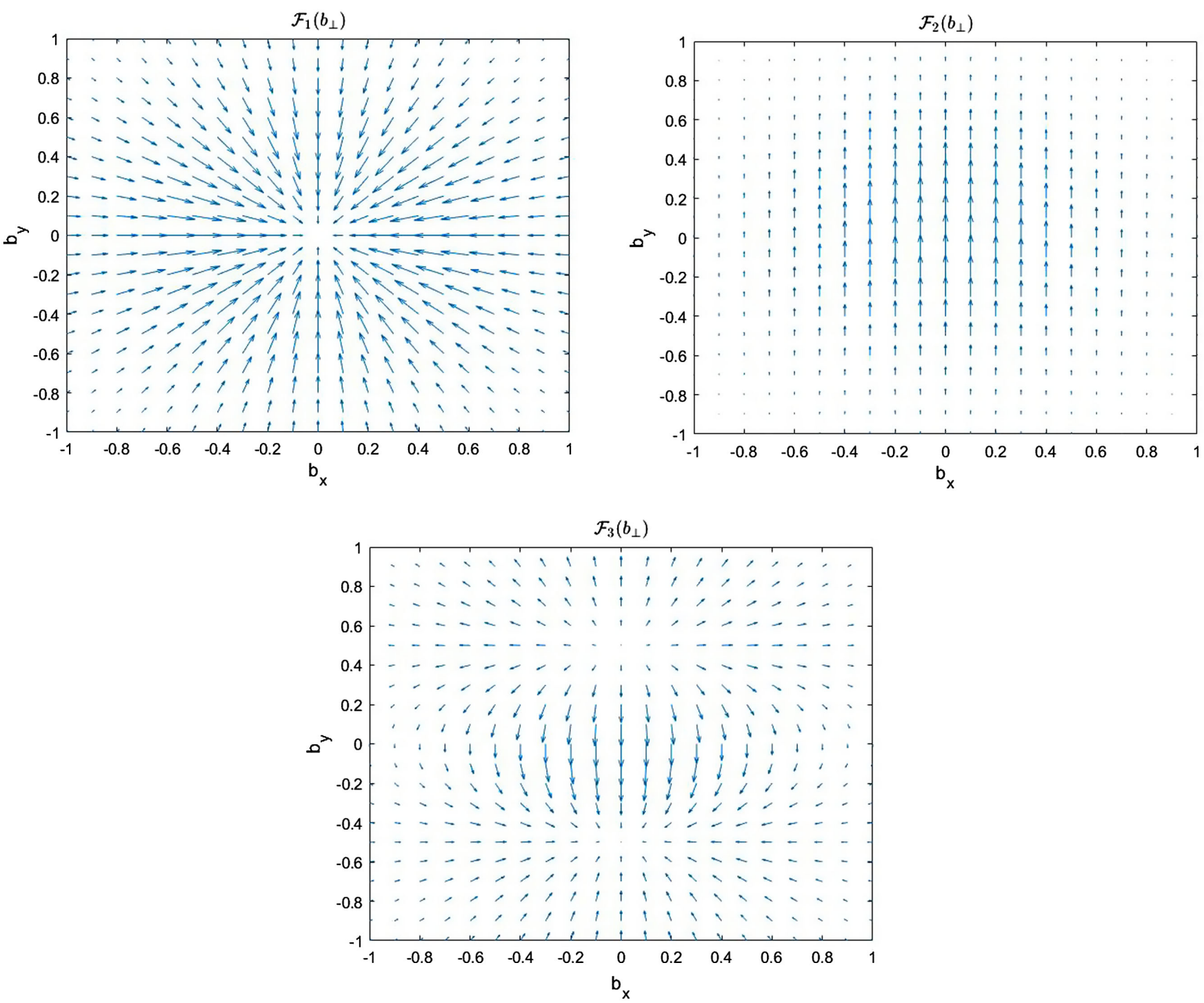

FIG. 1. Transverse force fields obtained from the three form factors, $\Phi_{1}, \Phi_{2}$ and $\Phi_{3}$ in Eq. (16). The orientations of the force fields $\mathcal{F}_{2}$ and $\mathcal{F}_{3}$ are for a nucleon polarized in the $x$-direction.

in terms of form factors. The way to parametrize $W$ closely follows the procedure outlined in Ref. [7]. In short, we assume the following ansatz for the matrix element $W$,

$$
W^{\mu, \nu \lambda}=\bar{u}\left(p^{\prime}, \lambda^{\prime}\right) \Gamma^{\mu, \nu \lambda}\left(p, p^{\prime}\right) u(p, \lambda),
$$

where $\Gamma$ is a general Dirac matrix depending on the initial and final nucleon momenta, $p$ and $p^{\prime}$, respectively. After decomposing $\Gamma$ into the sixteen basis matrices, $\left\{1, \gamma_{5}, \gamma^{\mu}\right.$, $\left.\gamma^{\mu} \gamma_{5}, i \sigma^{\mu \nu}\right\}$, the coefficients in that decomposition are parametrized in terms of the four momenta $p^{\mu}$ and $p^{\prime \mu}$, along with form factors that depend on $t=\Delta^{2}=\left(p^{\prime}-p\right)^{2}$. A parametrization with a minimal number of form factors is obtained by applying parity, time reversal, and hermiticity constraints, as well as Gordon identities.

For general Lorentz indices the matrix elements of the operator $\bar{q}(0) \gamma^{\mu} i g G^{\nu \lambda}(0) q(0)$ can be parametrized in terms of eight form factors [8]. However, for the transverse force distribution, we are only interested in the matrix elements of $\bar{q}(0) \gamma^{+} i g G^{+i}(0) q(0)$, which can be parametrized in terms of five form factors, $\Phi_{1}(t), \ldots, \Phi_{5}(t)$, as

$$
\begin{aligned}
\left\langle p^{\prime}, \lambda^{\prime}\left|\bar{q}(0) \gamma^{+} i g G^{+i}(0) q(0)\right| p, \lambda\right\rangle= & \bar{u}\left(p^{\prime}, \lambda^{\prime}\right)\left\{\frac{1}{M^{2}}\left[P^{+} \Delta_{\perp}^{i}-P^{\perp} \Delta^{+}\right)\right] \gamma^{+} \Phi_{1}(t)+\frac{P^{+}}{M} i \sigma^{+i} \Phi_{2}(t) \\
& \left.+\frac{1}{M^{3}} i \sigma^{+\Delta}\left[P^{+} \Delta_{\perp}^{i} \Phi_{3}(t)-P^{\perp} \Delta^{+} \Phi_{4}(t)\right]+\frac{P^{+} \Delta^{+}}{M^{3}} i \sigma^{i \Delta} \Phi_{5}(t)\right\} u\left(p^{\prime}, \lambda\right) .
\end{aligned}
$$


Here, $i$ corresponds to a transverse index $i=x, y$.

Combining Eq. (12) with Eq. (16) gives the spatial distributions of the force fields described by each form factor,

$$
\begin{aligned}
\mathcal{F}_{\lambda^{\prime} \lambda}^{i}\left(\mathbf{b}_{\perp}\right)= & \frac{i}{\sqrt{2} P^{+}} \int \frac{\mathrm{d}^{2} \boldsymbol{\Delta}_{\perp}}{(2 \pi)^{2}} \mathrm{e}^{-i \mathbf{b}_{\perp} \cdot \boldsymbol{\Delta}_{\perp}} \bar{u}\left(p^{\prime}, \lambda^{\prime}\right) \\
& \times\left[\frac{P^{+} \Delta_{\perp}^{i}}{M^{2}} \gamma^{+} \Phi_{1}\left(-\Delta_{\perp}^{2}\right)+\frac{P^{+}}{M} i \sigma^{+i} \Phi_{2}\left(-\Delta_{\perp}^{2}\right)\right. \\
& \left.+\frac{P^{+} \Delta_{\perp}^{i}}{M^{3}} i \sigma^{+\Delta} \Phi_{3}\left(-\Delta_{\perp}^{2}\right)\right] u(p, \lambda) .
\end{aligned}
$$

As for $q\left(x, b_{\perp}\right)$ in Eq. (1), the limit $\xi \rightarrow 0$ is necessary to develop a position space interpretation. Therefore, the $\Delta^{2}$ dependence of the form factors reduces to a $\Delta_{\perp}^{2}$ dependence in Eq. (17). Since $\Delta^{+}=0$ for $\xi=0$, the form factors $\Phi_{4}$, $\Phi_{5}$ and the second term in the coefficient of $\Phi_{1}$ in Eq. (16) do not appear in Eq. (17).

The force fields resulting from a Gaussian toy model for the form factors are depicted in Fig. 1.

Since $\bar{q}(0) \gamma^{+} g G^{+i}(0) q(0)$ is not sensitive to the polarization of quarks, the form factors describe forces on unpolarized quarks. Our comments on each term in Eq. (17) are as follows:

(i) The first term involving $\bar{u}\left(p^{\prime}, \lambda^{\prime}\right) \gamma^{+} u(p, \lambda)$ in Eq. (17) is diagonal in the helicities $\lambda, \lambda^{\prime}$ and therefore not sensitive to the nucleon polarization. Thus the Fourier transform of $\Delta_{\perp}^{i} \Phi_{1}$ yields the distribution of the axially symmetric radial force acting on unpolarized quarks in an unpolarized nucleon $\left(\mathcal{F}_{1}\left(\mathbf{b}_{\perp}\right)\right)$.

(ii) The second term in (17) involving $\bar{u}\left(p^{\prime}, \lambda^{\prime}\right) i \sigma^{+i}$ $u(p, \lambda)$ requires a nucleon helicity flip and it is thus sensitive to the transverse polarization of the nucleon. Therefore a Fourier transform of $\Phi_{2}$ describes the transverse force acting on unpolarized quarks in a transversely polarized nucleon and leads to the spatial distribution of the Sivers force $\left(\mathcal{F}_{2}\left(\mathbf{b}_{\perp}\right)\right)$.

(iii) The third term in (17) involving $\bar{u}\left(p^{\prime}, \lambda^{\prime}\right) i \sigma^{+\Delta} u(p, \lambda)$ also requires a nucleon helicity flip and depends on the transverse nucleon polarization as well. The position dependence described by a Fourier transform of $\Phi_{3}$ is similar to the transverse Lorentz force $\vec{v} \times \vec{B}$ for a charged particle moving through a magnetic dipole field $\left(\mathcal{F}_{3}\left(\mathbf{b}_{\perp}\right)\right)$.

\section{SUMMARY AND DISCUSSION}

Taking $x^{2}$ moments of twist-3 PDFs provides information about forward matrix elements of local quark-gluonquark correlators that have a very intuitive interpretation as the average transverse force acting on the active quark in a DIS experiment after absorbing the virtual photon. Similarly, $x^{2}$ moments of twist-3 GPDs yield nonforward matrix elements of the same local quark-gluon-quark correlator that appears in $x^{2}$ moments of twist-3 PDFs.

We have shown that by taking a Fourier transform of these nonforward matrix elements, one can determine how the transverse force depends on the impact parameter, $\mathbf{b}_{\perp}$.

Even though twist-3 GPDs are difficult to extract from experiment, the relevant matrix elements can also be obtained from lattice QCD calculations. The related form factors in Eq. (16) can be extracted by considering the nonforward matrix elements of the same operator that is used to calculate $d_{2}$ [9].

\section{ACKNOWLEDGMENTS}

This work was partially supported by the DOE under Grant No. DE-FG03-95ER40965 (F. A. and M. B.), and within the framework of the TMD Topical Collaboration.
[1] M. Burkardt, Phys. Rev. D 62, 071503 (2000); 66, 119903(E) (2002).

[2] M. Burkardt, Phys. Rev. D 88, 114502 (2013).

[3] K. Kanazawa, Y. Koike, A. Metz, D. Pitonyak, and M. Schlegel, Phys. Rev. D 93, 054024 (2016).

[4] S. Wandzura and F. Wilczek, Phys. Lett. 72B, 195 (1977).

[5] E. V. Shuryak and A. I. Vainshtein, Nucl. Phys. B201, 141 (1982).
[6] R. L. Jaffe, Comments Nucl. Part. Phys. 19, 239 (1990).

[7] S. Meissner, A. Metz, and M. Schlegel, J. High Energy Phys. 08 (2009) 056.

[8] Aslan, Burkardt, and Schlegel (to be published).

[9] M. Gockeler, R. Horsley, D. Pleiter, P. E. L. Rakow, A. Schafer, G. Schierholz, H. Stuben, and J. M. Zanotti, Phys. Rev. D 72, 054507 (2005). 